\title{
High prevalence of vitamin $D$ deficiency in patients with spinal cord injury: a 1 year longitudinal study
}

\author{
S. Wong ${ }^{1,2,3}$, I. Gainullina ${ }^{1}$, A. Graham ${ }^{1}$, S. P. Hirani ${ }^{2}$ and A. Forbes ${ }^{3}$ \\ ${ }^{1}$ National Spinal Injuries Centre, Stoke Mandeville Hospital, Aylesbury, ${ }^{2}$ Health Service Research, City University \\ London and ${ }^{3}$ Centre of Gastroenterology and Clinical Nutrition, University College London, London
}

Previous studies reported that the prevalence of hypovitaminosis D is increased amongst spinal cord injured (SCI) individuals. ${ }^{(1)}$ This could possibly due to decreased mobility, prolonged institutionalisation and reduced exposure to sunlight following SCI. Hypovitaminosis D can be influenced by factors such the seasons. This study aimed to (1) evaluate 25 -hydroxyvitamin D concentrations in SCI patients admitted to a UK SCI centre over four seasons and (2) to assess the characteristics of vitamin D deficiency in this patient group. 245 adults (mean age: 50.1 years, $22.8 \%$ female) with SCI (58.6\% tetraplegia; $48.6 \%$ complete SCI) were studied prospectively during April 2012 to February 2013. Data was collected by two trained professionals (research doctor and dietitian) from individual patient notes using a standardised questionnaire. Vitamin D levels $(\mathrm{nmol} / \mathrm{l})<50 ;<80$ and $>80$ were defined as deficient; insufficient and normal, respectively. Almost all (93.1\%) SCI patients were vitamin D insufficient, $67.8 \%$ had vitamin D deficiency. Hypovitaminosis D was associated with undernutrition risk2 $(p=0.013)$, onset of SCI $(p=0.029)$, severity of SCI: complete SCI $(p=0.014)$ and non-summer seasons $(p=0.02)$. Multivariate logistic regression identified severity of SCI as an independent factor for hypovitaminosis D (OR: 3.53; 95\% CI: 1.31-9.51). Hypovitaminosis D was found to be less common in summer when compared to Autumn, Winter and Spring. $(54.5 \%, 66.7 \%, 78.1 \%, 65 \%)$ The present study shows hypovitaminosis D is common. Complete SCI is an independent risk factor. Strategies for systematic screening and treatment of hypovitaminosis D need to be refined and implemented. Given the severe loss of quality of life for these patients, a cost-effective, reliable therapy would appear to be highly desirable. Further study of vitamin D supplementation and body composition in SCI patients is warranted.

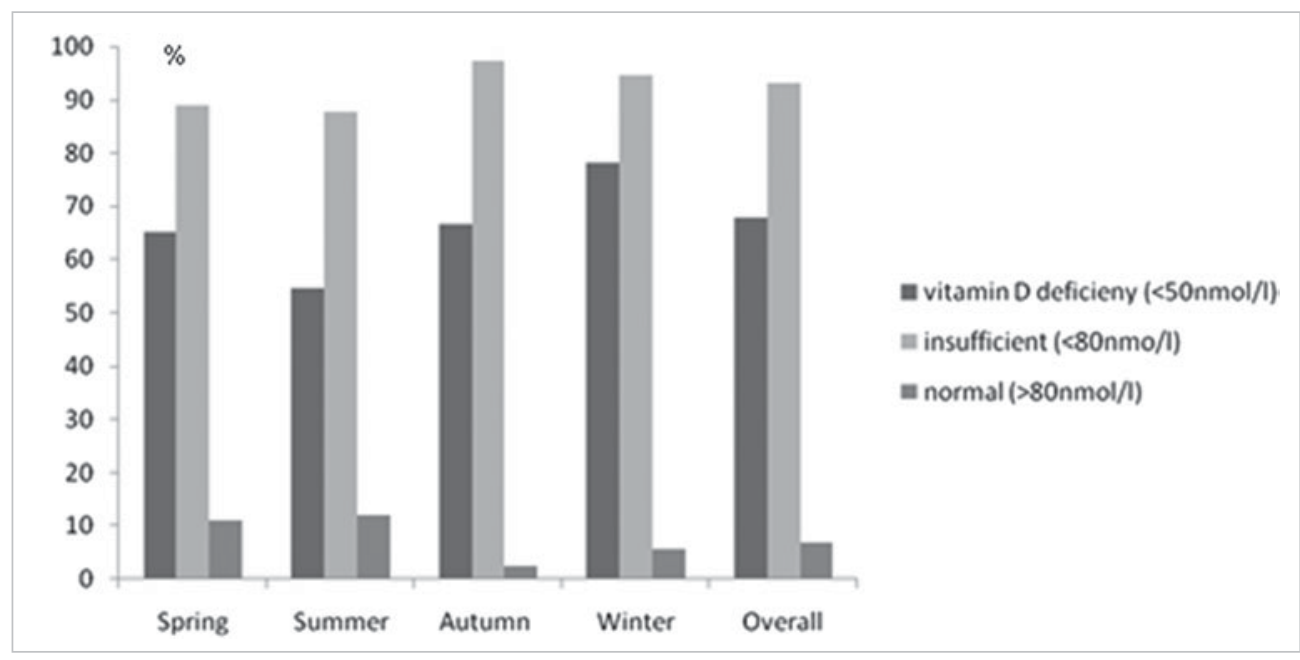

The authors would like to thanked Veronica Moore (National Spinal Injuries Centre, UK) for her assistance in facilitating the data collection and inputting data in the database.

1. Zafeiris et al. (2012) Spine $J$ 12, 304-312.

2. Wong S et al. (2012) Eur J Clin Nutr 66, 382-387. 